Modern Physics Letters A

Vol. 20, No. 5 (2005) 373

(C) World Scientific Publishing Company

\title{
ERRATUM
}

\section{CP NONINVARIANCE AND AN EFFECTIVE COSMOLOGICAL CONSTANT: THE ENERGY DENSITY IN A PSEUDOSCALAR FIELD WHICH ARISES FROM A COSMOLOGICAL, SPONTANEOUSLY-BROKEN CHIRAL SYMMETRY}

\author{
[Mod. Phys. Lett. A, Vol. 19, No. 39 (2004) 2899-2908]
}

S. Barshay and G. Kreyerhoff

In Refs. 13, the first page number should read 167, and the third page number should read 369 .

Supposing that both very light $b$ particles and very heavy $\phi$ particles constitute a part of the dark matter, then exchange of a very light $b$ particle between a pair of $b$, a pair of $\phi$ and a $\phi$ - $b$ pair (assumed to have very small, intrinsic relative velocity), gives rise to attractive long-range potentials. Taking the ratio of the strength of the new Yukawa potential for a $b$-pair, within the range $\sim 1 / m_{b}$, to the gravitational potential for a $\phi$-pair, we obtain $1 / 64 \pi\left(\phi_{c} / M_{\mathrm{P}}\right)^{2} \sim 1$, independent of the parameter $\lambda$. The possibly greater coherent effect of the new potential between concentrations of large numbers of dark-matter particles originates in the possibility that the number density of very light $b$ can be greater than that of very massive $\phi$. It is possible that a present number density of $b$ particles could originate in production by a time-varying gravitational field ${ }^{8}$ over a time interval during the early rapid expansion of the universe.

We thank Prof. P. J. E. Peebles for sending us stimulating remarks about his own concern for a possible long-range force affecting only dark-matter particles, and the effects upon the formation of, or absence of, structure. 